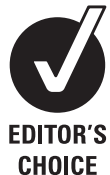

CHOICE

\section{See Editorial, p 1537}

${ }^{1}$ The Hatter Cardiovascular Institute, University College London, London, UK ${ }^{2}$ Medical Statistics Unit, London School of Hygiene and Tropical Medicine, London, UK

${ }^{3}$ The Heart Hospital, University College London Hospital, London, UK

${ }^{4}$ UCL Institute of Child Health, Great Ormond Street Hospital, London, UK

\section{Correspondence to}

Professor Derek M Yellon, The Hatter Cardiovascular Institute University College London, 67 Chenies Mews, London WC1E 6HX, UK;

hatter.institute@ucl.ac.uk

Accepted 21 June 2011

\title{
Effect of erythropoietin as an adjunct to primary percutaneous coronary intervention: a randomised controlled clinical trial
}

\author{
Andrew J Ludman, ${ }^{1}$ Derek M Yellon, ${ }^{1}$ Jonathan Hasleton, ${ }^{1}$ Cono Ariti, ${ }^{2}$ \\ Girish Ganesha Babu, ${ }^{1}$ Edney Boston-Griffiths, ${ }^{1}$ Vinod Venugopal, ${ }^{1}$ Malcolm Walker, ${ }^{3}$ \\ Diana Holdright, ${ }^{3}$ Howard Swanton, ${ }^{3}$ Tom Crake, ${ }^{3}$ David Brull, ${ }^{3}$ James C Moon, ${ }^{3}$ \\ Rajesh Puranik, ${ }^{4}$ Vivek Mutharangu, ${ }^{4}$ Andrew Taylor, ${ }^{4}$ Derek J Hausenloy ${ }^{1}$
}

\begin{abstract}
Objective The acute administration of high-dose erythropoietin (EPO) on reperfusing ischaemic myocardium has been reported to halve myocardial infarct (MI) size in preclinical studies, but its effect in ST elevation myocardial infarction patients undergoing primary percutaneous coronary intervention (PPCI) remains unknown. We investigated whether high-dose EPO administered as an adjunct to PPCI reduces MI size. Design Double-blinded, randomised, placebo-controlled. Setting Single tertiary cardiac centre.

Patients Fifty-one ST elevation myocardial infarction patients undergoing PPCl.
\end{abstract}

Interventions Patients were randomly assigned to receive either a single intravenous bolus of EPO (50 $000 \mathrm{IU}$ ) prior to $\mathrm{PPCl}$ with a further bolus given $24 \mathrm{~h}$ later $(n=26)$ or placebo $(n=25)$.

Main outcome measures $\mathrm{Ml}$ size measured by $24 \mathrm{~h}$ area under the curve troponin $T$ and cardiac magnetic resonance imaging performed on day 2 and at 4 months. Results EPO treatment failed to reduce MI size (troponin $T$ area under the curve: $114.6 \pm 78 \mu \mathrm{g} / \mathrm{ml}$ EPO vs $100.8 \pm 68 \mu \mathrm{g} / \mathrm{ml}$ placebo; infarct mass by cardiac magnetic resonance: $33 \pm 16 \mathrm{~g}$ EPO vs $25 \pm 16 \mathrm{~g}$ placebo; both $\mathrm{p}>0.05)$. Unexpectedly, EPO treatment doubled the incidence of microvascular obstruction $182 \%$ EPO vs $47 \%$ placebo; $p=0.02$ ) and significantly increased indexed left ventricular (LV) end-diastolic volumes $\left(84 \pm 10 \mathrm{ml} / \mathrm{m}^{2}\right.$ EPO vs $73 \pm 13 \mathrm{ml} / \mathrm{m}^{2}$ placebo; $\mathrm{p}=0.003)$, indexed LV end-systolic volumes $\left(41 \pm 9 \mathrm{ml} / \mathrm{m}^{2}\right.$ EPO vs $35 \pm 11 \mathrm{ml} / \mathrm{m}^{2}$ placebo; $\left.p=0.035\right)$ and indexed myocardial mass $\left(89 \pm 16 \mathrm{~g} / \mathrm{m}^{2}\right.$ EPO vs $79 \pm 11 \mathrm{~g} / \mathrm{m}^{2}$ placebo; $\left.p=0.03\right)$. At 4 months, there were no significant differences between groups.

Conclusions High-dose EPO administered as an adjunct to PPCI failed to reduce $\mathrm{Ml}$ size. In fact, EPO treatment was associated with an increased incidence of microvascular obstruction, LV dilatation and increased LV mass.

Clinical Trial Registration Information http://public. ukcrn.org.uk/search/StudyDetail.aspx?StudyID $=4058$ Unique Identifier=Study ID 4058.

\section{INTRODUCTION}

Despite recent advances in primary percutaneous coronary intervention (PPCI), the morbidity and mortality of ST-elevation myocardial infarction (STEMI) patients remain significant. This may be due, in part, to the presence of lethal myocardial reperfusion injury, a phenomenon in which the reperfusion of ischaemic myocardium induces further cardiomyocyte death, thereby limiting the beneficial effects of myocardial reperfusion by PPCI. ${ }^{1}$ Therefore, novel cardioprotective agents capable of reducing lethal myocardial reperfusion injury are required to realise the full benefits of reperfusion therapy, limit myocardial infarct (MI) size, preserve cardiac function and improve clinical outcomes in these patients.

There is considerable evidence that the cytokine erythropoietin (EPO) exerts pleiotropic effects beyond that of haematopoiesis, which include both neuroprotection and cardioprotection. ${ }^{2}$ In preclinical studies, a number of beneficial pleiotropic effects of EPO have been reported, including stimulation of neovascularisation, beneficial peri-infarct and myocardial remodelling as well as direct cellular protective effects, which, by activating a number of cell survival pathways, render the myocyte more resistant to ischaemia-reperfusion injury. $^{2}$ The acute administration of high-dose EPO at the time of reperfusion has been reported in animal models to reduce MI size by approximately $50 \%{ }^{2}$ Initial pilot studies in patients have demonstrated safety with high-dose EPO. ${ }^{3}{ }^{4}$ Whether EPO is beneficial in STEMI patients when administered as an adjunct to PPCI remains unknown.

\section{METHODS}

\section{Patient selection}

This study received local Ethics Committee approval and was carried out in accordance with the University College London Hospital Trust guidelines. Between July 2007 and August 2009, we conducted a randomised, double-blinded, placebocontrolled trial of STEMI patients referred to a single tertiary cardiac centre for PPCI. We obtained written informed consent from all patients who entered the study. Patients were included if they presented for PPCI within $12 \mathrm{~h}$ of onset of symptoms and had the following ECG criteria ( $\geq 2 \mathrm{~mm}$ ST-elevation in chest ECG leads, $\geq 1 \mathrm{~mm}$ ST-elevation in contiguous limb leads or new-onset left bundle branch block), were under 80 years old with a single-vessel culprit lesion and had Thrombolysis in Myocardial Infarction grade 0 and Rentrop collateral grade 0 on coronary angiography. Exclusion criteria included abnormal renal 
function (serum creatinine $>120 \mu \mathrm{mol} / \mathrm{l}$ ), known thromboembolic disorder, malignant disease, multivessel disease, cardiogenic shock and cardiac arrest. Patients were excluded from cardiac magnetic resonance (CMR) imaging if they had any metal implants which rendered CMR unsafe or if they were claustrophobic.

\section{Procedures}

Eligible consenting patients were randomly allocated to receive either EPO treatment or placebo prior to PPCI. A computergenerated blocked randomisation list was prepared in advance of the trial. Treatment allocation was undertaken by a Clinical Research Fellow (not involved with assessing clinical outcomes or the PPCI procedure) using sealed opaque envelopes. The patient, the PPCI operator and the assessor of clinical outcomes were blinded to the treatment allocation.

The EPO treatment arm comprised 50000 IU of rhEPO $\beta$ (NeoRecormon, Roche Ltd., Welwyn Garden City, UK) in $10 \mathrm{ml}$ $0.9 \%$ saline, whereas the placebo comprised $10 \mathrm{ml} 0.9 \%$ saline. Either was administered via a peripheral intravenous cannula over $2 \mathrm{~min}$ prior to any device crossing the occluded vessel during PPCI, with an additional rhEPO $\beta$ dose or placebo administered via the same route $24 \mathrm{~h}$ following PPCI. Once reconstituted, the solutions appeared identical. The dose of rhEPO $\beta$ was obtained from a previously published proof-ofconcept clinical study which suggested that rhEPO $\beta$ (33000 IU) administered daily for 3 days (total dose 99000 IU) may be beneficial in patients with stroke. ${ }^{3}$ PPCI was undertaken according to the cardiologists' preference, although thrombolytics and adenosine were not used. Blood samples for troponin Tand creatine kinase- $\mathrm{MB}$ (CK-MB) were taken prior to PPCI and 6, 12 and 24 h post-procedure. Blood samples for haemoglobin, haematocrit, platelet count, prothrombin time and renal function were taken at baseline and at 2 days post-PPCI.

The primary endpoint was MI size as determined by late gadolinium enhancement (LGE) CMR. Secondary endpoints included $24 \mathrm{~h}$ area under the curve (AUC) serum CK-MB and troponin $\mathrm{T}$, myocardial salvage index and CMR-determined left ventricular (LV) volumes, mass and ejection fraction and the presence of microvascular obstruction (MVO).

\section{CMR imaging}

CMR imaging was performed at a median of 2 days from PPCI (range 1-6 days) and repeated at 4 months using a $1.5 \mathrm{~T}$ scanner (Avanto-Siemens, Erlangen, Germany). LV function and volumes were assessed by standard steady-state free precession technique. Consecutive short-axis views were obtained by encompassing the LV from base to apex; vertical and horizontal long-axis views were acquired. Typical image parameters were as follows: echo time (TE) $1.16 \mathrm{~ms}$, repetition time (TR) $2.73 \mathrm{~ms}$, flip angle $65^{\circ}$, matrix $144 \times 192$, slice thickness $7 \mathrm{~mm}$, gap $3 \mathrm{~mm}$. A velocityencoded aortic flow map was acquired to confirm LV volumes. Rest myocardial perfusion images were evaluated with a firstpass technique using a T1-weighted multishot gradient echoplanar inversion-recovery sequence (TR $2.6 \mathrm{~ms}$, TE $1.1 \mathrm{~ms}$, inversion time (TI) $200 \mathrm{~ms}$, flip angle $12^{\circ}$, slice thickness $10 \mathrm{~mm}$ ). Three short-axis slices (basal, mid-cavity and apex) were obtained injecting $0.2 \mathrm{mmol} / \mathrm{kg}$ gadolinium (Dotarem; Guerbet SA, Paris, France) at $2 \mathrm{ml} / \mathrm{s}$ followed by a $20 \mathrm{ml}$ saline flush into an anterocubital vein. Early gadolinium enhancement images were acquired 1-2 min after gadolinium injection with a fixed TI of $440 \mathrm{~ms}$. Two-dimensional slices in LV short axis were imaged with no interslice gap (TR $7.0 \mathrm{~ms}$, TE $4.9 \mathrm{~ms}$, flip angle $23^{\circ}$ ). LGE images were acquired in long- and short-axis views with a segmented inversion-recovery fast gradient echo sequence $10 \mathrm{~min}$ after the contrast injection. Sequence parameters were as follows: TR $8.9 \mathrm{~ms}$, TE $4.9 \mathrm{~ms}$, flip angle $25^{\circ}$, slice thickness $7 \mathrm{~mm}$, gap $3 \mathrm{~mm}$. The TI was optimised to null normal myocardium.

\section{CMR analysis}

All CMR images were analysed by an experienced CMR reader (A.J.L. or J.H.). All LV volume data were independently and blindly analysed by both readers. LV ejection fraction (LVEF) and end-systolic and end-diastolic volumes and mass were calculated from segmentation of the LV and indexed to body surface area. The rest perfusion, early gadolinium enhancement and LGE images were assessed qualitatively for the presence or absence of MVO as an area of hypoenhancement on the early and LGE scans (see figure 1). MI mass was measured by manual segmentation of areas of LGE and analysed by concordance between the two readers. In case of discordance, blinded review by a level III accredited CMR reader (J.C.M. or D.J.H.) was performed. Analysis was performed using OsiriX (version 3.5.1) software. Interobserver variability was calculated.

\section{Determining the myocardial salvage index}

When assessing the efficacy of a reperfusion treatment strategy, it is essential to express MI size (IS) as a percentage of the area at risk (AAR). In this study, the AAR was quantified using both
Figure 1 Representative images of an initial CMR scan displaying early gadolinium enhancement revealing evidence of microvascular obstruction (left panel*) and late gadolinium enhancement (right panel) depicting a transmural myocardial infarct (arrow) with a core of microvascular obstruction $(*)$.
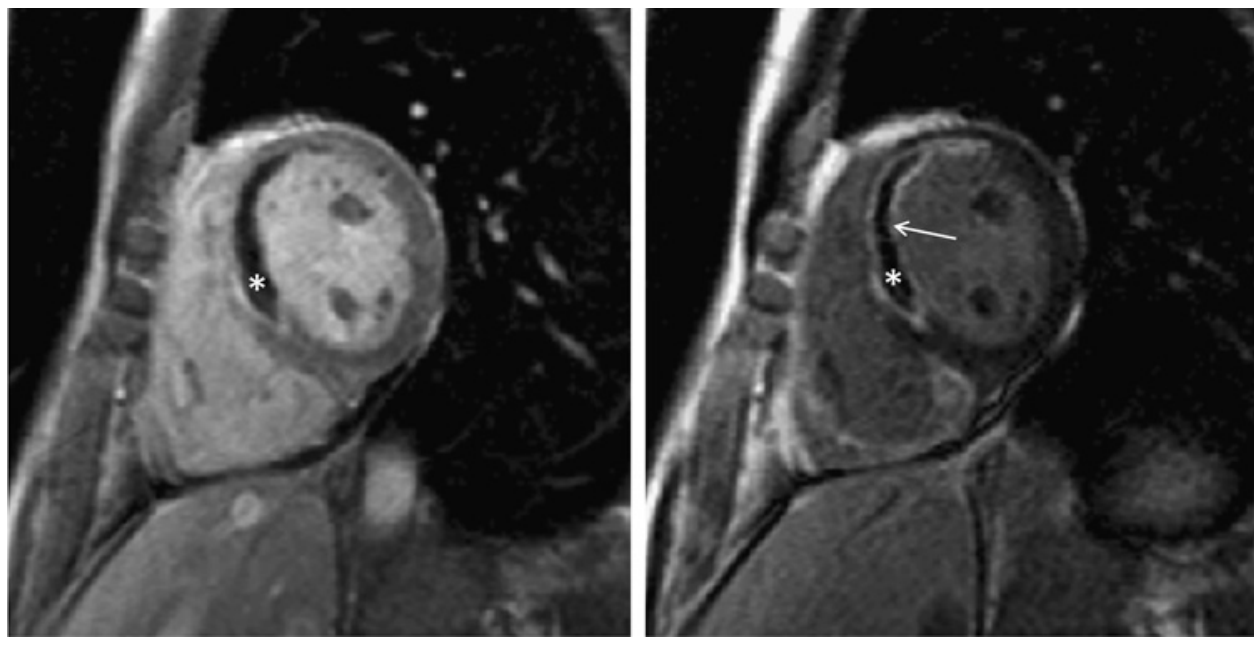
coronary angiography (modified Bypass Angioplasty Revascularisation Investigation (BARI) and Alberta Provincial Project for Outcome Assessment in Coronary Heart Disease (APPROACH) jeopardy scores) ${ }^{5}$ and the acute CMR scan (infarct endocardial surface area (Infarct-ESA))..$^{5}$ As a measure of the AAR, Infarct-ESA has been validated against the BARI and APPROACH scores $^{5}$ and T2-weighted imaging of myocardial oedema. ${ }^{6}$ Myocardial salvage index was calculated as follows: (AAR-IS)/AAR.

\section{Statistical analysis}

Descriptive statistics were computed to summarise the relevant features of the data. An unpaired t test was used to test the differences in means in the EPO versus placebo groups for continuous data, and 95\% CIs were calculated for the difference of the means between the EPO and placebo groups. The assumptions of the $t$ test were examined using residuals analysis. A sensitivity analysis was performed where individual patients had Studentised residuals greater than two SDs by omitting these patients from the analysis and examining the impact to the mean difference and 95\% CIs. A Bland-Altman plot was used to calculate the difference and limits of agreement between the two observers for each continuous clinical measurement. The number of measurements falling outside the limits of agreement was used as a heuristic guide to agreement. A paired $t$ test of the measurements was used to formally test that the difference of the means of the two measurements was zero. In addition, the reliability (intraclass correlation) was computed for each continuous clinical measurement. A $\kappa$ statistic was calculated to measure the observer agreement for categorical clinical measurements. For categorical data, a Z-test of proportions was used to test the equality of the proportion of patients in the EPO group versus the control group. An approximate $95 \%$ CI for the difference in the proportions in the two groups was also calculated.

At study conception, a sample size of at least 44 patients in total was determined based on the following assumptions: (a) a 36\% reduction in $72 \mathrm{~h}$ total CK release from 326095 to 208984 arbitrary units; (b) power of at least $80 \%$; (c) an SD of 135846 arbitrary units and (d) significance declared at the two-sided 5\% level. ${ }^{7}$ However, due to the nonspecificity of total CK measurements along with the advent and validation of $\mathrm{CK}-\mathrm{MB}$ and troponin $\mathrm{T}$, these were measured instead. However, with the advent of newly presented data, we were able to retrospectively validate the sample size of the study using the endpoint of CMR-assessed MI size. The infarct-limiting effects of ciclosporin A were investigated in a similar cohort of patients. A sample size of at least 40 patients in total was determined based on the following assumptions: (a) a $20 \%$ reduction in mass of $\mathrm{MI}$ as measured by CMR scan from $46 \mathrm{~g}$ to $37 \mathrm{~g}$; (b) power of at least $80 \%$; (c) an SD of $10 \mathrm{~g}$ and (d) significance declared at the two-sided $5 \%$ level. ${ }^{8}$

All analyses were done with Stata V.11 or SPSS statistical software V.15.0.

\section{RESULTS}

Figure 2 shows the trial profile. One hundred and ten patients were screened for inclusion in the study, with 51 patients randomised to receive either placebo $(n=25)$ or $\operatorname{rhEPO} \beta(n=26)$. The treatment group characteristics are summarised in table 1. There was good agreement using the three different scores to measure the AAR. Table 2 summarises the AAR scores and the main results. MI size was assessed by $24 \mathrm{~h}$ AUC serum troponin $\mathrm{T}(114.6 \mu \mathrm{g} / \mathrm{ml}$ EPO vs $100.8 \mu \mathrm{g} / \mathrm{ml}$ placebo (95\% CI -29.7 to 57.3))

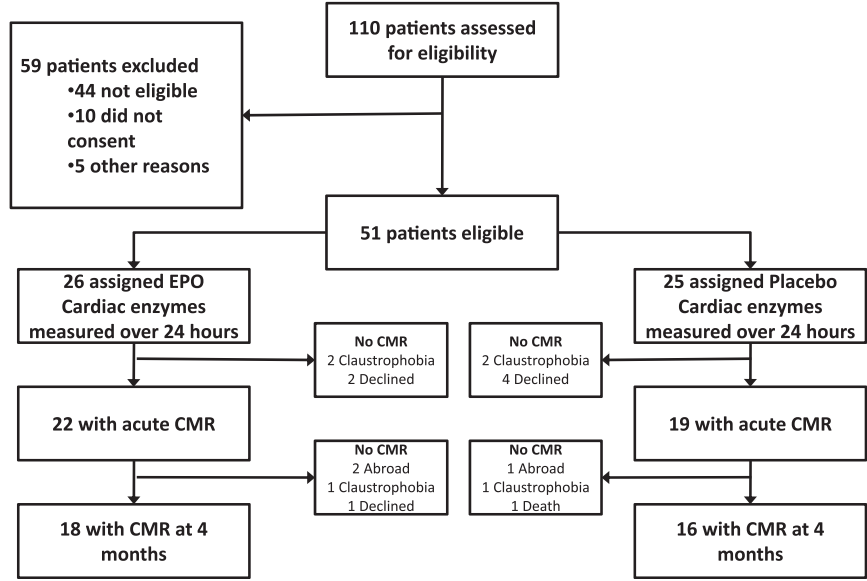

Figure 2 Trial recruitment profile.

and CK-MB (4682 U/1 EPO vs $3649 \mathrm{U} / 1$ placebo (95\% CI -563 to 2629)) and by the initial (33 g EPO vs 25 g placebo (95\% CI -2.8 to 17.7$)$ ) and follow-up (18 g EPO vs $12 \mathrm{~g}$ placebo (95\% CI -0.6 to 11.2$)$ ) CMR scans.

Unexpectedly, at the initial CMR scan, the presence of MVO was almost doubled in the EPO-treated group when compared to placebo. Furthermore, there was an increase in indexed LV end-systolic and end-diastolic volumes and mass in the EPOtreated group compared to placebo. There were no significant differences between the groups in terms of the LV stroke volume index and LVEF and the myocardial salvage index. There were no significant differences in the CMR endpoints on the 4-month follow-up scan, although there was a trend to increased IS and reduced LVEF in the EPO-treated group compared to placebo.

There was good to excellent agreement between observers with the intraclass correlation ranging from 0.7 to 0.95 .

All serious adverse events were reported to the safety committee and investigated. There was no significant difference in adverse events between the two groups. There were no deaths in the eligible patients randomised to EPO. There was one death in the placebo group during a subsequent PCI to the nonindex vessel. There were five admissions to hospital for evaluation of chest pain in the EPO group and one in the placebo group, none of which were deemed a new acute coronary syndrome. There were two planned PCI procedures in both groups and one planned coronary artery bypass graft surgery in the EPO group. One LV apical thrombus was found at 4 months in the EPO group.

\section{DISCUSSION}

The major findings of this double-blinded, randomised clinical trial are that the administration of high-dose $\operatorname{rhEPO} \beta$ as an adjunct to PPCI had no beneficial effects in terms of MI size, myocardial salvage index or LVEF. In fact, the findings suggest that rhEPO $\beta$ may actually be detrimental in this setting, as it almost doubled the incidence of MVO. Furthermore, it was associated with acute LV dilatation and an acute increase in myocardial mass. On the 4-month follow-up CMR scan, there were no significant differences between the treatment groups.

It is well established that in STEMI patients successfully treated with PPCI, the presence of MVO is associated with worse clinical outcomes. ${ }^{9} 10 \mathrm{MVO}$ can be detected in about $30 \%-40 \%$ of PPCI patients using echocardiography ${ }^{11}$ or CMR, ${ }^{10}{ }^{12}$ even in the presence of Thrombolysis in Myocardial Infarction grade III coronary artery flow post-PPCI. In our study, in patients treated 
Table 1 Patient characteristics

\begin{tabular}{|c|c|c|}
\hline & EPO $(n=26)$ & Placebo $(n=25)$ \\
\hline Age, mean years & $55.5(12.8)$ & $61(10.0)$ \\
\hline Men & $23(88)$ & $21(84)$ \\
\hline Hypercholesterolaemia & $7(30)$ & $13(50)$ \\
\hline Diabetes mellitus & $3(12)$ & $2(8)$ \\
\hline Hypertension & $10(39)$ & $13(50)$ \\
\hline Family history of IHD & $4(17)$ & $5(21)$ \\
\hline Previous myocardial infarction & $2(9)$ & $1(4)$ \\
\hline Previous angina & $1(4)$ & $1(4)$ \\
\hline Respiratory disease & $2(9)$ & $1(4)$ \\
\hline \multicolumn{3}{|l|}{ Smoking status } \\
\hline Never & $10(38)$ & $9(36)$ \\
\hline Ex-smoker & $5(19)$ & $4(16)$ \\
\hline Current & $11(43)$ & $12(48)$ \\
\hline \multicolumn{3}{|l|}{ Medication at presentation (\%) } \\
\hline Aspirin & $5(19)$ & $5(20)$ \\
\hline Clopidogrel & 0 & 0 \\
\hline ACE-I/ARB & $4(15)$ & $6(24)$ \\
\hline$\beta$-Blocker & $1(4)$ & $2(8)$ \\
\hline CCB & $3(12)$ & $1(4)$ \\
\hline Statin & $4(15)$ & $6(24)$ \\
\hline Nitrate & $1(4)$ & 0 \\
\hline Sulphonylurea & $1(4)$ & $2(8)$ \\
\hline Metformin & $2(8)$ & $2(8)$ \\
\hline PPI & $1(4)$ & $1(4)$ \\
\hline \multicolumn{3}{|l|}{ Blood result at presentation } \\
\hline Haemoglobin (g/dl) & $14.7(1.2)$ & $14.2(1.1)$ \\
\hline Haematocrit & $0.42(0.03)$ & $0.41(0.03)$ \\
\hline Platelet count $\left(10^{9} / l\right)$ & $256(53)$ & $254(76)$ \\
\hline Prothrombin time (s) & $10.7(0.7)$ & $10.5(0.5)$ \\
\hline Creatinine $(\mu \mathrm{mol} / \mathrm{l})$ & $83(15)$ & $79(14)$ \\
\hline \multicolumn{3}{|l|}{ Blood result day 2} \\
\hline Haemoglobin (g/dl) & $13.6(1.6)$ & $13.6(1.6)$ \\
\hline Haematocrit & $0.40(0.04)$ & $0.39(0.04)$ \\
\hline Platelet count $\left(10^{9} / \mathrm{I}\right)$ & $217(53)$ & $234(83)$ \\
\hline Creatinine $(\mu \mathrm{mol} / \mathrm{l})$ & $83(14)$ & $82(15)$ \\
\hline \multicolumn{3}{|l|}{ Culprit vessel } \\
\hline LAD & $10(38)$ & $13(52)$ \\
\hline $\mathrm{LCx}$ & $7(27)$ & $4(16)$ \\
\hline $\mathrm{RCA}$ & $9(35)$ & $8(32)$ \\
\hline Symptom to PPCl time, mean (min) & $224(104)$ & $257(156)$ \\
\hline Door to balloon time, mean (min) & $56(24)$ & $39(16)$ \\
\hline \multicolumn{3}{|l|}{ Medication during PPCI } \\
\hline Aspirin & $26(100)$ & $25(100)$ \\
\hline Clopidogrel & $26(100)$ & $25(100)$ \\
\hline Heparin & $26(100)$ & $25(100)$ \\
\hline Opiate & $15(58)$ & $13(52)$ \\
\hline Nitrate & $13(50)$ & $16(64)$ \\
\hline Abciximab & $25(96)$ & $23(92)$ \\
\hline Metoclopramide & $7(27)$ & $10(40)$ \\
\hline Atropine & $4(15)$ & $7(28)$ \\
\hline Mean TIMI flow before PPCI (grade) & 0 & 0 \\
\hline Mean TIMI flow after PPCI (grade) & 2.92 & 2.96 \\
\hline Rentrop grade (collateralisation) & 0 & 0 \\
\hline Thrombectomy device used & $9(35)$ & $1(4)$ \\
\hline Lesion predilated & $25(96)$ & $23(92)$ \\
\hline Direct stent to lesion & $1(4)$ & $2(8)$ \\
\hline Bare metal stent & $17(65)$ & $16(64)^{*}$ \\
\hline Drug-eluting stent & $9(35)$ & $10(40)^{*}$ \\
\hline \multicolumn{3}{|l|}{ Mean arterial pressure, $\mathrm{mm} \mathrm{Hg}$} \\
\hline Pre-PCl & $110(14)$ & $111(22)$ \\
\hline Post-PCl & $97(16)$ & $95(22)$ \\
\hline Day 2 & $93(14)$ & $95(11)$ \\
\hline Follow-up & $102(15)$ & $103(17)$ \\
\hline
\end{tabular}

Table 1 Continued

\begin{tabular}{lll}
\hline & EPO (n=26) & Placebo (n=25) \\
\hline $\begin{array}{l}\text { Median length of hospital stay, days } \\
\text { (range) }\end{array}$ & $3.0(2-6)$ & $3.5(2-10)$ \\
$\begin{array}{l}\text { Medication on discharge (\%) } \\
\text { Aspirin }\end{array}$ & $25(96)$ & $25(100)$ \\
Clopidogrel & $26(100)$ & $25(100)$ \\
ACE-I/ARB & $25(96)$ & $25(100)$ \\
$\beta$-Blocker & $23(88)$ & $20(80)$ \\
Statin & $26(100)$ & $25(100)$ \\
PPI & $3(13)$ & $1(5)$ \\
Medication at follow-up (\%) & $16(89)$ & $14(88)$ \\
Aspirin & $12(67)$ & $12(75)$ \\
Clopidogrel & $17(94)$ & $13(81)$ \\
ACE-I/ARB & $14(78)$ & $12(75)$ \\
$\beta$-Blocker & $15(83)$ & $13(81)$ \\
Statin & $1(6)$ & $1(6)$ \\
PPI & $2(1-4)$ & $2(1-6)$ \\
Median time to acute CMR scan (range) & $126(116-160)$ & $131(106-163)$ \\
Median time to follow-up CMR scan \\
(range)
\end{tabular}

with rhEPO $\beta$, the incidence of MVO detected by the initial CMR scan was dramatically increased from $47 \%$ in placebo to $82 \%$, a finding which might be expected to be associated with worse clinical outcomes. Importantly, factors which are known to impact on the development of MVO such as the presence of comorbid conditions, antiplatelet and anticoagulant therapy and MI size were not significantly different between the two treatment groups. MVO results in poor myocardial perfusion despite epicardial coronary artery revascularisation, and its development has been attributed to a variety of factors including distal embolisation, endothelial dysfunction, leucocyte migration and plugging and platelet aggregation. ${ }^{13}$ The mechanism underlying the increased incidence of MVO following PPCI in the rhEPO $\beta$ treated group in our study is unknown but may be attributed to the increased platelet reactivity and the prothrombotic effects which have been described with rhEPO therapy in healthy subjects and a number of different disease states. ${ }^{14}{ }^{15}$ Interestingly, high-dose rhEPO (intravenous $400 \mathrm{IU} / \mathrm{kg}$ given daily for 3 days) significantly blunted the aspirin-mediated increase in bleeding time when compared with placebo in healthy volunteers, although no effect was observed on the clopidogrel-mediated increase in bleeding time. ${ }^{16}$

The explanation for the significantly increased myocardial mass (calculated from the myocardial volume) observed on the initial CMR scan in patients treated with rhEPO $\beta$ is unknown. It may have been due to enhanced myocardial oedema associated with more severe myocardial reperfusion injury, as suggested by the greater incidence of $\mathrm{MVO}$ in the rhEPO $\beta$-treated patients. In this respect, an increased indexed myocardial mass has been noted by CMR 1 week following an acute myocardial infarction in a previous study. ${ }^{17}$ The acute LV dilatation noted in patients treated with rhEPO $\beta$ may well be a compensatory response to greater myocardial injury, where an increase in preload provokes LV dilatation in an attempt to maintain the LV stroke volume.

Initial pilot investigations of rhEPO in human settings of stroke, ${ }^{3}$ non-STEMI ${ }^{18}$ and STEMI ${ }^{4}$ have demonstrated safety and 
Table 2 Clinical outcomes

\begin{tabular}{|c|c|c|c|c|}
\hline & $\begin{array}{l}\mathrm{EPO}, \mathrm{n}=26 \\
\text { (22 with acute } \mathrm{CMR} \text { ) }\end{array}$ & $\begin{array}{l}\text { Placebo, } n=25 \\
\text { (19 with acute CMR) }\end{array}$ & Difference $(95 \% \mathrm{CI})$ & p Value \\
\hline \multicolumn{5}{|c|}{ Initial blood results, myocardial area at risk and CMR scan } \\
\hline $24 \mathrm{~h}$ troponin $\mathrm{T}$ AUC $(\mu \mathrm{g} / \mathrm{l})$ & 114.6 & 100.8 & $13.81(-29.69$ to 57.31$)$ & 0.526 \\
\hline 24 h CK-MB AUC (U/I) & 4682.3 & 3649.1 & $1033.2(-562.7$ to 2629.1$)$ & 0.199 \\
\hline BARI score (\%) & 29 & 33 & $-3.8(-8.6$ to 1.0$)$ & 0.12 \\
\hline APPROACH score (\%) & 30 & 31 & $-0.8(-6.8$ to 5.2$)$ & 0.8 \\
\hline Infarct-ESA by CMR (\%) & 28 & 27 & $0.8(-5.4$ to 7.0$)$ & 0.8 \\
\hline Myocardial infarct mass $(\mathrm{g})$ & 32.8 & 25.4 & $7.46(-2.76$ to 17.69$)$ & 0.148 \\
\hline Myocardial salvage index & 0.38 & 0.47 & $-0.09(-0.28$ to 0.10$)$ & 0.333 \\
\hline Microvascular obstruction (\%) & 81.8 & 47.4 & $34.4(6.8-62.1)$ & 0.020 \\
\hline LVEDVi $\left(\mathrm{ml} / \mathrm{m}^{2}\right)$ & 84.4 & 73.0 & $11.40(4.05-18.75)$ & 0.003 \\
\hline LVESVi $\left(\mathrm{ml} / \mathrm{m}^{2}\right)$ & 41.3 & 34.6 & $6.77(0.45-13.09)$ & 0.036 \\
\hline LVSVi $\left(\mathrm{ml} / \mathrm{m}^{2}\right)$ & 43.1 & 38.4 & $4.63(-0.44$ to 9.70$)$ & 0.072 \\
\hline LVMi $\left(g / \mathrm{m}^{2}\right)$ & 89.2 & 79.4 & $9.78(0.94-18.63)$ & 0.031 \\
\hline LVEF (\%) & 51.3 & 53.2 & $-1.87(-7.40$ to 3.66$)$ & 0.499 \\
\hline \multicolumn{5}{|l|}{ 4-month follow-up CMR endpoints } \\
\hline LVEDVi $\left(\mathrm{ml} / \mathrm{m}^{2}\right)$ & 88.1 & 84.4 & $3.74(-10.11$ to 17.58$)$ & 0.586 \\
\hline LVESVi $\left(\mathrm{ml} / \mathrm{m}^{2}\right)$ & 42.7 & 36.4 & $6.30(-6.60$ to 19.21$)$ & 0.327 \\
\hline LVSVi $\left(\mathrm{ml} / \mathrm{m}^{2}\right)$ & 45.4 & 48.0 & $-2.56(-8.24$ to 3.11$)$ & 0.364 \\
\hline LVMi $\left(\mathrm{g} / \mathrm{m}^{2}\right)$ & 82.4 & 75.0 & $7.33(-5.00$ to 19.56$)$ & 0.235 \\
\hline LVEF (\%) & 52.6 & 59.1 & $-6.46(-13.94$ to 1.01$)$ & 0.088 \\
\hline Myocardial infarct mass $(\mathrm{g})$ & 17.7 & 12.4 & $5.31(-0.57$ to 11.19$)$ & 0.075 \\
\hline \multicolumn{5}{|l|}{ Clinical outcomes } \\
\hline Death & 0 & 1 & & \\
\hline Stroke & 0 & 0 & & \\
\hline Unplanned revascularisation & 1 & 0 & & \\
\hline Planned revascularisation & 2 & 2 & & \\
\hline Emergency admission to hospital & 5 & 1 & & \\
\hline LV thrombus & 1 & 0 & & \\
\hline
\end{tabular}

a potential trend to benefit in terms of reduction in IS. These pilot studies were initiated following a considerable number of preclinical studies demonstrating a range of beneficial effects in small animal infarct models (reviewed by Riksen et $\mathrm{al}^{2}$ ), with protective actions thought to be mediated via the EPO receptor which has been demonstrated in human cardiomyocytes. ${ }^{19}$ Interestingly, the infarct-limiting effects of EPO as reperfusion therapy in larger animal studies of ischaemia-reperfusion injury such as sheep ${ }^{20}$ and porcine ${ }^{21}$ have been negative.

Subsequently, several studies have investigated high-dose rhEPO as a novel cytoprotective agent in STEMI. The administration of rhEPO $\beta$ (30000 IU) prior to tenecteplase thrombol$y$ sis in STEMI patients failed to find any difference in cardiac enzyme release or any difference in major adverse cardiac events. ${ }^{22}$ A pilot study of $30 \mathrm{PPCI}$ patients has reported that the administration of rhEPO $\alpha$ (33000 IU daily for 3 days) reduced $\mathrm{CK}-\mathrm{MB}$ release but failed to limit $\mathrm{MI}$ size on a CMR scan performed within 3 days of PPCI. ${ }^{23}$ In that study, the effect of rhEPO $\alpha$ on CMR-detected MVO was not reported. A lower dosing regimen of $12000 \mathrm{U}$ rhEPO $\beta$ following PPCI for STEMI in 36 patients $^{24}$ was unable to demonstrate a difference in the primary endpoint of LVEF by radionucleotide scintigraphy between groups.

Regenerate Vital Myocardium by Vigorous Activation of Bone Marrow Stem Cells (REVIVAL-3) reported that treatment with rhEPO $\beta$ (33 333 IU daily for 3 days) starting immediately after successful PPCI in 138 patients made no difference to LVEF measured at 6 months by CMR and that there was a trend to adverse cardiovascular outcomes (death, recurrent myocardial infarction, infarct-related artery revascularisation and stroke). ${ }^{25}$ The HEBE- 3 study ${ }^{26}$ recruited 529 patients undergoing PPCI and randomised to $60000 \mathrm{IU}$ of rhEPO $\alpha$ or placebo within $3 \mathrm{~h}$ following reperfusion. There was no significant difference in the primary endpoint of LVEF at 6 weeks by radionucleotide ventriculography. The authors reported secondary endpoints of a favourable safety profile and a statistically significant reduction in adverse events, although total numbers of events were small.

Further studies in other clinical settings of ischaemiareperfusion injury including coronary artery bypass graft surgery ${ }^{27}$ and stroke ${ }^{15}$ have also been negative, with significantly increased adverse events reported in patients with stroke treated with rhEPO $\alpha$ within 6 h of stroke onset.

In our study and those above, the failure of rhEPO to reduce MI size in PPCI patients is in conflict with the preclinical data reporting $50 \%$ reductions in $\mathrm{MI}$ size in rat, rabbit and dog models of ischaemia-reperfusion injury, with EPO administered at time of reperfusion. ${ }^{2}$ We administered the rhEPO $\beta$ prior to any device crossing the occluded coronary artery in order to ensure that high serum levels of EPO were present at the moment of reperfusion, aiming to prevent myocyte injury caused at this time. The dose was the same total dose as that in a promising pilot study in stroke and has been shown to generate high serum EPO levels ${ }^{3}$; a study of a lower dose of rhEPO is also negative, ${ }^{24}$ and the majority of successful animal studies have been performed with very high doses of between 350 and $5000 \mathrm{IU} / \mathrm{kg}^{2}{ }^{2}$ Discordant findings between preclinical animal studies and the clinical translation of novel cardioprotective strategies has been a recurring issue, the causes of which 
have been highlighted in several recent articles. ${ }^{1} 28$ Specifically, the proinflammatory and prothrombotic conditions associated with an acute myocardial infarction in a middle-aged patient with comorbidities such as diabetes, dyslipidaemia and hypertension are not reproduced by experimental coronary artery occlusion in disease-free juvenile small- to medium-size animal models.

Furthermore, there has been a recent challenge to the pleiotropic abilities of EPO and doubt cast over the presence of biologically active EPO receptor in tissues not involved in erythropoeisis $^{29}$; however, this paper has been criticised by a large body of researchers for overstating its conclusions ${ }^{30}$ and not providing enough evidence for which to discount the work of a large body of other investigators. Thus, the debate as to the exact abilities of EPO continues; however, our study and others have been unable to demonstrate a benefit of acute high-dose EPO in the setting of STEMI, and a number of studies have raised concerns about adverse events. Further studies should be performed with caution, given the current uncertainty about the potential mechanism for an increase in associated adverse events. Further work continues in elucidating the protective pathways of EPO in the preclinical setting and exploring EPO analogues (such as carbamylated EPO), which may provide protective benefits without the apparent associated side effects.

In summary, we have demonstrated that the administration of high-dose rhEPO $\beta$ as an adjunct to PPCI failed to reduce MI size or improve cardiac function. In fact, its use may actually be detrimental in that it was associated with a doubling in the incidence of MVO, acute LV dilatation and increased myocardial mass. This study highlights the importance of CMR as a technique for assessing the safety and efficacy of novel reperfusion therapies. The findings from the current study should be considered, alongside those of other recently published studies, in the design of future studies investigating the use of high-dose rhEPO in patients with acute myocardial infarction.

Acknowledgements We thank the British Heart Foundation (Program Grant RG/ 03/007) for their ongoing funding and support. We offer our grateful thanks to all the patients and staff, particularly Drs Clare Dollery, Dominic Rogers, Alamgir Kabir, Anoop Shetty, Paul Kotwinski, Vanessa Cobb, Vineet Wadhera, A.B. Gopalamurugan and Costas O'Mahoney at The Heart Hospital for their assistance during this study.

Funding The work was supported by Roche Ltd. which kindly supplied the rhEPO $\beta$ (NeoRecormon), but it played no role in conception, conduct or analysis of this study. This work was undertaken at University College London Hospital/University College London which received a proportion of funding from the Department of Health's National Institute of Health Research Biomedical Research Centres funding scheme.

Competing interests None declared.

Patient consent Obtained.

Ethics approval This study was conducted with the approval of the University College London R\&D department.

Provenance and peer review Not commissioned; externally peer reviewed.

\section{REFERENCES}

1. Yellon DM, Hausenloy DJ. Myocardial reperfusion injury. N Engl J Med 2007; 357:1121-35.

2. Riksen NP, Hausenloy DJ, Yellon DM. Erythropoietin: ready for prime-time cardioprotection. Trends Pharmacol Sci 2008;29:258-67.

3. Ehrenreich $\mathbf{H}$, Hasselblatt M, Dembowski C, et al. Erythropoietin therapy for acute stroke is both safe and beneficial. Mol Med 2002:8:495-505.
4. Lipsic $\mathbf{E}$, van der MP, Voors AA et al. A single bolus of a long-acting erythropoietin analogue darbepoetin alfa in patients with acute myocardial infarction: a randomized feasibility and safety study. Cardiovasc Drugs Ther 2006;20:135-41.

5. Ortiz-Perez JT, Meyers SN, Lee DC, et al. Angiographic estimates of myocardium at risk during acute myocardial infarction: validation study using cardiac magnetic resonance imaging. Eur Heart $J$ 2007;28:1750-8.

6. Wright J, Adriaenssens T, Dymarkowski S, et al. Quantification of myocardial area at risk with T2-weighted CMR: comparison with contrast-enhanced CMR and coronary angiography. JACC Cardiovasc Imaging 2009:2:825-31.

7. Staat $\mathbf{P}$, Rioufol G, Piot $\mathrm{C}$, et al. Postconditioning the human heart. Circulation 2005; 112:2143-8.

8. Piot C, Croisille P, Staat $P$, et al. Effect of cyclosporine on reperfusion injury in acute myocardial infarction. N Engl J Med 2008;359:473-81.

9. Wu KC, Zerhouni EA, Judd RM, et al. Prognostic significance of microvascular obstruction by magnetic resonance imaging in patients with acute myocardial infarction. Circulation 1998:97:765-72.

10. Hombach V, Grebe 0 , Merkle N, et al. Sequelae of acute myocardial infarction regarding cardiac structure and function and their prognostic significance as assessed by magnetic resonance imaging. Eur Heart $J$ 2005;26:549-57.

11. Ito $\mathbf{H}$, Maruyama A, Iwakura $\mathrm{K}$, et al. Clinical implications of the 'no reflow' phenomenon. A predictor of complications and left ventricular remodelling in reperfused anterior wall myocardial infarction. Circulation 1996;93:223-8.

12. Bogaert J, Kalantzi M, Rademakers FE, et al. Determinants and impact of microvascular obstruction in successfully reperfused ST-segment elevation myocardial infarction. Assessment by magnetic resonance imaging. Eur Radiol 2007:17:2572-80.

13. Lee CH, Tse HF. Microvascular obstruction after percutaneous coronary intervention Catheter Cardiovasc Interv 2009:75:369-77.

14. Lippi G, Franchini M, Favaloro EJ. Thrombotic complications of erythropoiesisstimulating agents. Semin Thromb Hemost 2010;36:537-49

15. Ehrenreich H, Weissenborn K, Prange H, et al; EPO Stroke Trial Group. Recombinan human erythropoietin in the treatment of acute ischemic stroke. Stroke 2009;40: e647-56.

16. Tang YD, Rinder HM, Katz SD. Effects of recombinant human erythropoietin on antiplatelet action of aspirin and clopidogrel in healthy subjects: results of a doubleblind, placebo-controlled randomized trial. Am Heart J 2007;154:494.e1-7.

17. Schroeder AP, Houlind K, Pederson EM, et al. Serial magnetic resonance imaging of global and regional left ventricular remodelling during 1 year after acute myocardia infarction. Cardiology 2001:96:106-14.

18. Liem A, van de Woestijne AP, Bruijns E, et al. Effect of EPO administration on myocardial infarct size in patients with non-STE acute coronary syndromes; results from a pilot study. Int J Cardiol 2007;131:285-7.

19. Depping R, Kawakami K, Ocker H, et al. Expression of the erythropoietin receptor in human heart. J Thorac Cardiovasc Surg 2005;130:877-8.

20. Olea FD, Janavel GV, De Lorenzi A, et al. High-dose erythropoietin has no long-term protective effects in sheep with reperfused myocardial infarction. J Cardiovasc Pharmacol 2006:47:736-41.

21. Toma C, Letts DP, Tanabe M, et al. Positive effect of darbepoetin on peri-infarction remodeling in a porcine model of myocardial ischemia-reperfusion. J Mol Cell Cardiol 2007:43:130-6.

22. Binbrek AS, Rao NS, Al Khaja N, et al. Erythropoietin to augment myocardial salvage induced by coronary thrombolysis in patients with ST segment elevation acute myocardial infarction. Am J Cardiol 2009;104:1035-40.

23. Ferrario $\mathbf{M}$, Arbustini $\mathrm{E}$, Massa $\mathbf{M}$, et al. High-dose erythropoietin in patients with acute myocardial infarction: a pilot, randomised, placebo-controlled study. Int J Cardiol 2011:147:124-31.

24. Ozawa T, Toba K, Suzuki H, et al; EPO/AMI-I Pilot Study Researchers. Single-dose intravenous administration of recombinant human erythropoietin is a promising treatment for patients with acute myocardial infarction-randomized controlled pilot trial of EPO/AMl-1 study. Circ J 2010;74:1415-23.

25. Ott I, Schulz S, Mehilli J, et al: REVIVAL-3 Study Investigators. Erythropoietin in patients with acute ST-segment elevation myocardial infarction undergoing primary percutaneous coronary intervention: a randomized, double-blind trial. Circ Cardiovasc Interv 2010;3:408-13

26. Voors AA, Belonje AM, Zijlstra F, et al; HEBE III Investigators. A single dose of erythropoietin in ST-elevation myocardial infarction. Eur Heart J 2010;31:2593-600.

27. Mocini D, Muso P, Guendouz E, et al. Endogenous erythropoietin and a single bolus of 40,000 IU of epoetin $\alpha$ do not protect the heart from ischaemia-reperfusion injury during extracorporeal circulation for cardiac surgery. Perfusion 2008:23:187-92.

28. Ludman AJ, Yellon DM, Hausenloy DJ. Cardiac preconditioning for ischaemia: lost in translation. Dis Model Mech 2010;3:35-8.

29. Swift $\mathbf{S}$, Ellison $A R$, Kassner $P$, et al. Absence of functional EpoR expression in human tumor cell lines. Blood 2010;115:4254-63.

30. Ghezzi P, Bernaudinb M, Bianchic R, et al. Erythropoietin: not just about erythropoiesis. Lancet 2010;375:2142. 


\section{CORRESPONDENCE}

Transradial primary PCl: this is the time for awareness

To the Editor Things take some time to change, but we are on the right path. I must respectfully disagree, however, with the conclusions reached by Patterson and Foale on the performance of transradial primary percutaneous coronary interventions (PCIs). ${ }^{1}$ After a brief history of the technique and despite an overview of the enormous advantages derived from its use (fewer bleedings, lower incidence of composite end point of death, stroke and myocardial infarction), they reach the conclusion that urgent PCI should not be performed via the transradial route.

In our opinion, the advantages of this route of intervention unfold during primary PCI. We will try to prove the following: coronary interventions are performed with a 6-Fr introducer; hence, the radial route is practicable in $>90 \%$ of cases. During primary $\mathrm{PCI}$, it is common to use more potent antithrombotic drugs (ie, abciximab, prasugrel) that are associated with a higher bleeding risk. In this journal, Hetherington et $a l^{2}$ recently published the results of a study performed in a single high-volume centre that involved 1051 patients undergoing primary PCI. Procedures performed with the transradial route, apart from warranting fewer bleedings if compared with the transfemoral route, were discovered to reduce inhospital major adverse cardiovascular and cerebrovascular events, door-to-balloon and symptom-to-balloon time, contrast load and radiation dose. Interestingly, if operators at the beginning of the study preferred the transfemoral route in $80 \%$ of cases, at the end, only $20 \%$ of patients were treated through the femoral artery. In case of cardiogenic shock, the transradial route should be preferred because it avoids dual femoral puncture and interference with the balloon counterpulsation.

We radialists are well aware that this route of intervention requires advanced expertise and a steep learning curve. With a high level of expertise, however, it becomes apparent that it does not increase procedural and fluoroscopy time and why access site crossover rate is very low. Moreover, costs for the healthcare system are lower and, more importantly, patient outcome is similar or superior without any inconvenience. ${ }^{3}$
Patients are happy to walk out of the cath lab after having undergone elective procedures. To conclude, the advantages are considerable in the case of primary PCI.

\section{Bernardo Cortese}

Correspondence to Dr Bernardo Cortese, Interventional Cardiology, Cliniche Gavazzeni, 24121, Bergamo, Italy; bcortese@gmail.com

Competing interests None declared.

Provenance and peer review Not commissioned; internally peer reviewed.

Published Online First 13 September 2011

Heart 2011;97:1812. doi:10.1136/heartjnl-2011-300597

\section{REFERENCES}

1. Patterson T, Foale RA. If the radial artery is the new standard of care in primary percutaneous coronary intervention, why is most intervention done by the femoral approach? Heart 2011;97:521-2.

2. Hetherington SL, Adam Z, Morley R, et al. Primary percutaneous coronary intervention for acute ST-segment elevation myocardial infarction: changing patterns of vascular access, radial versus femoral artery. Heart 2009;95:1612-18.

3. Amoroso G, Kiemeneij F. Transradial access for primary percutaneous coronary intervention: the next standard of care? Heart 2010;96:1341-4.

The Authors' reply We would like to thank Dr Cortese for his letter. ${ }^{1}$ As previously stated, we agree that the radial approach has been associated with fewer access site complications. $^{2}$ In his correspondence, Cortese refers to the study by Hetherington et $a l^{3}$ who in fact demonstrated no statistically significant difference in in-hospital mortality and major adverse cardiovascular and cerebrovascular events after Bonferroni correction and, interestingly, found no difference in vascular complication rates (radial $0.5 \%$ (3/571) vs femoral 1.3\% (6/481), $\mathrm{p}=0.315)$ with a higher incidence of procedural failure via the radial approach despite operator selection (OR 19.8 (95\% CI 5.75 to 68.3), $p<0.001)$. Cortese is correct in emphasising the reduced inpatient stay and reduction in cost associated with radial angioplasty, ${ }^{2}$ and in the primary percutaneous coronary intervention setting, Hetherington et al did demonstrate a trend towards this, albeit in the presence of operator selection bias as it was not a randomised controlled trial, which, in practice, could have been translated into a preference for radial approach in a clinically more stable patient group.

With regards to cardiogenic shock, this cohort is often excluded from trials and we would be interested to hear Dr Cortese's views on this specific group. Our experience, to date, continues to support the femoral approach given ease of access in an emergency, dual-site venous access and intraaortic balloon pump insertion. Although we were intrigued that Cortese performs radial angioplasty with femoral intra-aortic balloon pump, the evidence points toward dualaccess-site management being the most problematic

\section{Tiffany Patterson, R A Foale}

Department of Cardiology, Imperial College Healthcare NHS Trust, London, UK

Correspondence to Dr Tiffany Patterson, Department of Cardiology, Imperial College Healthcare NHS Trust, London, UK; tiffany.patterson@imperial.nhs.uk

\section{Competing interests None.}

Provenance and peer review Commissioned; internally peer reviewed.

Heart 2011;97:1812. doi:10.1136/heartjnl-2011-300766

\section{REFERENCES}

1. Cortese B. Transradial primary PCl: this is the time for awareness. Heart 2011;97:1812.

2. Patterson T, Foale RA. If the radial artery is the new standard of care in primary percutaneous coronary intervention, why is most intervention done by the femoral approach? Heart 2011;97:521-2.

3. Hetherington SL, Adam Z, Morley R, et al. Primary percutaneous coronary intervention for acute ST-segment elevation myocardial infarction: changing patterns of vascular access, radial versus femoral artery. Heart 2009;95:1612-18.

\section{CORRECTION}

doi:10.1136/hrt.2011.223867corr1

Ludman AJ, Yellon DM, Hasleton J, et al. Effect of erythropoietin as an adjunct to primary percutaneous coronary intervention: a randomised controlled clinical trial Heart 2011;97:1560-5. One of the authors' surname was spelt incorrectly in this paper and should read as follows: Vivek Muthurangu. 